\title{
A CONNECTION BETWEEN A GENERAL CLASS OF SUPERPARABOLIC FUNCTIONS AND SUPERSOLUTIONS
}

\author{
RIIKKA KORTE, TUOMO KUUSI, AND MIKKO PARVIAINEN
}

\begin{abstract}
We show to a general class of parabolic equations that every bounded superparabolic function is a weak supersolution and, in particular, has derivatives in a Sobolev sense. To this end, we establish various comparison principles between super- and subparabolic functions, and show that a pointwise limit of uniformly bounded weak supersolutions is a weak supersolution.
\end{abstract}

\section{INTRODUCTION}

We study a connection between two different classes of supersolutions to the equation

$$
\frac{\partial u}{\partial t}=\operatorname{div} \mathcal{A}(x, t, \nabla u)
$$

where $\mathcal{A}$ is strictly monotonic, satisfies the $p$-growth conditions, $p>2 n /(n+$ 2 ), as well as the well-known Carathéodory-type conditions. Weak supersolutions to (1.1) are defined by testing with smooth functions and they are assumed to belong to a parabolic Sobolev space. In contrast, $\mathcal{A}$-superparabolic functions are defined as lower semicontinuous functions obeying the comparison principle. We show in Theorem 5.8 that every uniformly bounded $\mathcal{A}$-superparabolic function is a weak supersolution and, in particular, has derivatives in a Sobolev sense. Since $\mathcal{A}$-superparabolic functions are not required to have any derivatives, this is somewhat surprising. Moreover, examples show that unbounded $\mathcal{A}$-superparabolic functions sometimes fail to be supersolutions.

In the proof of our main result, we approximate an $\mathcal{A}$-superparabolic function by a sequence of solutions to obstacle problems. We aim at deriving the desired properties for the limit from the corresponding properties for the sequence. To show that the approximating sequence converges pointwise, we use an elliptic-type comparison principle. By definition, $\mathcal{A}$-superparabolic functions satisfy the comparison in space-time boxes. We use the Schwarz alternating method in the parabolic setting to show in Lemma 4.5 of Section 4 (see also Lemma 4.6) that the comparison also holds in more general sets. In the nonlinear elliptic case, the method is applied, for example, in Granlund-Lindqvist-Martio [7]. In the proof of the elliptic comparison principle we also use a fact that a weak subsolution can be modified by an $\mathcal{A}$-parabolic function in a space-time cylinder so that it remains a weak subsolution. This and related results are proven in Section 3. Next we pass from pointwise convergence to a stronger convergence and show that limit

2000 Mathematics Subject Classification. 35K55, 35B50, 31A05. 
of a sequence of uniformly bounded weak supersolutions is a weak supersolution. We prove this highly useful property in Theorem 5.3 of Section 5 following Lindqvist and Manfredi [13]. The proof of the convergence result makes no difference between increasing and decreasing convergence.

Kinnunen and Lindqvist proved in [11] that bounded superparabolic functions to the evolutionary $p$-Laplace equation, $p \geq 2$, are supersolutions. Their proof utilizes approximated equations and a special convolution. In [13], Lindqvist and Manfredi proved, using the infimal convolution, that bounded viscosity supersolutions to the evolutionary $p$-Laplace equation, $p \geq 2$, have spatial gradients. Our proof employs basic tools and readily extends to a more general class of equations as such. Furthermore, the singular case when $p>2 n /(n+2)$ is also included.

Our results seem to be valuable in applications, for example, to nonlinear parabolic potential theory including Perron's method for general equations and the regularity of boundary points. For the evolutionary $p$-Laplace equation, Kilpeläinen and Lindqvist discuss these issues in [9]. Moreover, the methods established by Kinnunen and Lindqvist in [10] can be applied, together with our results, to the general equation: It turns out that unbounded $\mathcal{A}$-superparabolic functions belong to the lower Sobolev spaces.

Further applications include the existence of solutions to general initial value and measure data problems, cf. DiBenedetto-Herrero [5], BoccardoDall'Aglio-Gallouët-Orsina [3]. Indeed, the convergence result also implies the useful weak convergence of the non-negative Radon measures generated by supersolutions.

It is also worth noting that superparabolic functions to the evolutionary $p$-Laplace equation coincide with the viscosity supersolutions, as Juutinen, Lindqvist and Manfredi proved in [8].

\section{Preliminaries}

We begin with the notation and assumptions. In what follows, $Q$ will stand for a box

in $\mathbb{R}^{n}$, and the sets

$$
Q=\left(a_{1}, b_{1}\right) \times \ldots \times\left(a_{n}, b_{n}\right)
$$

$$
Q_{T}=Q \times(0, T), \quad Q_{t_{1}, t_{2}}=Q \times\left(t_{1}, t_{2}\right)
$$

in $\mathbb{R}^{n} \times \mathbb{R}$ are called space-time boxes. Let $U$ be an open set in $\mathbb{R}^{n}$. The parabolic boundary of a cylinder $U_{t_{1}, t_{2}}=U \times\left(t_{1}, t_{2}\right) \subset \mathbb{R}^{n} \times \mathbb{R}$ is

$$
\partial_{p} U_{t_{1}, t_{2}}=\left(\bar{U} \times\left\{t_{1}\right\}\right) \cup\left(\partial U \times\left(t_{1}, t_{2}\right]\right) .
$$

We define the parabolic boundary of a finite union of open cylinders $U_{t_{1}^{i}, t_{2}^{i}}^{i}$ as follows

$$
\partial_{p}\left(\cup U_{t_{1}^{i}, t_{2}^{i}}^{i}\right)=\left(\cup \partial_{p} U_{t_{1}^{i}, t_{2}^{i}}^{i}\right) \backslash\left(\cup U_{t_{1}^{i}, t_{2}^{i}}^{i}\right),
$$

If $D^{\prime}$ is a bounded open subset of $D$ and the closure of $D^{\prime}$ belongs to $D$, we denote $D^{\prime} \Subset D$.

Let $\Xi$ be an open set in $\mathbb{R}^{n} \times \mathbb{R}$. We assume that $\mathcal{A}: \Xi \times \mathbb{R}^{n} \mapsto \mathbb{R}^{n}$ is a monotonic Carathéodory function that satisfies $p$-growth bounds, i.e.

(1) $(x, t) \mapsto \mathcal{A}(x, t, \xi)$ is measurable for every $\xi \in \mathbb{R}^{n}$,

(2) $\xi \mapsto \mathcal{A}(x, t, \xi)$ is continuous for almost every $(x, t) \in \Xi$, 
(3) there exist constants $0<\alpha \leq \beta<\infty$ and $p>2 n /(n+2)$ such that

$$
\mathcal{A}(x, t, \xi) \cdot \xi \geq \alpha|\xi|^{p} \quad \text { and } \quad|\mathcal{A}(x, t, \xi)| \leq \beta|\xi|^{p-1},
$$

for almost every $(x, t) \in \Xi$ and every $\xi \in \mathbb{R}^{n}$,

(4) whenever $\left(x, t, \xi_{i}\right) \in \Xi \times \mathbb{R}^{n}, i=1,2$, and $\xi_{1} \neq \xi_{2}$,

$$
\left(\mathcal{A}\left(x, t, \xi_{1}\right)-\mathcal{A}\left(x, t, \xi_{2}\right)\right) \cdot\left(\xi_{1}-\xi_{2}\right)>0 .
$$

Let $U$ be a bounded open set in $\mathbb{R}^{n}$. As usual, $W^{1, p}(U)$ denotes the space of real-valued functions $f$ such that $f \in L^{p}(U)$ and the distributional first partial derivatives $\partial f / \partial x_{i}, i=1,2, \ldots, n$, exist in $U$ and belong to $L^{p}(U)$. We use the norm

$$
\|f\|_{1, p, U}=\left(\int_{U}|f|^{p} d x\right)^{1 / p}+\left(\int_{U}|\nabla f|^{p} d x\right)^{1 / p} .
$$

The Sobolev space with zero boundary values, $W_{0}^{1, p}(U)$, is the closure of $C_{0}^{\infty}(U)$ with respect to the Sobolev norm.

Weak sub- and supersolutions belong to a parabolic Sobolev space, which guarantees a priori summability of the function and its gradient. By the parabolic Sobolev space

$$
L^{p}\left(t_{1}, t_{2} ; W^{1, p}(U)\right),
$$

$t_{1}<t_{2}$, we mean the space of functions such that the function $x \mapsto u(x, t)$ belongs to $W^{1, p}(U)$ for almost every $t_{1}<t<t_{2}$ and the norm

$$
\left(\int_{t_{1}}^{t_{2}} \int_{U}|u(x, t)|^{p}+|\nabla u(x, t)|^{p} d x d t\right)^{1 / p}
$$

is finite. The definition for the space $L^{p}\left(t_{1}, t_{2} ; W_{0}^{1, p}(U)\right)$ is analogous.

Next we define weak solutions to equation (1.1).

Definition 2.3. Let $\Xi$ be an open set in $\mathbb{R}^{n} \times \mathbb{R}$. A function $u$ is a weak solution in $\Xi$, if $u \in L^{p}\left(t_{1}, t_{2}, W^{1, p}(U)\right)$ whenever $U_{t_{1}, t_{2}} \Subset \Xi$, and $u$ satisfies the integral equality

$$
\int_{t_{1}}^{t_{2}} \int_{U} \mathcal{A}(x, t, \nabla u) \cdot \nabla \phi d x d t-\int_{t_{1}}^{t_{2}} \int_{U} u \frac{\partial \phi}{\partial t} d x d t=0
$$

for all $\phi \in C_{0}^{\infty}\left(U_{t_{1}, t_{2}}\right)$. A function $u$ is a weak supersolution (subsolution), if $u \in L^{p}\left(t_{1}, t_{2}, W^{1, p}(U)\right)$ whenever $U_{t_{1}, t_{2}} \Subset \Xi$, and the expression above is non-negative (non-positive) for all non-negative $\phi \in C_{0}^{\infty}\left(U_{t_{1}, t_{2}}\right)$. Continuous weak solutions are called $\mathcal{A}$-parabolic functions.

We recall examples of equations for which some weak solutions are known. The standard example is the evolutionary $p$-Laplace equation

$$
\frac{\partial u}{\partial t}=\nabla \cdot\left(|\nabla u|^{p-2} \nabla u\right)
$$

In [2], Barenblatt found a self-similar weak solution, named after himself, to the evolutionary $p$-Laplace equation

$$
\mathcal{B}_{p}(x, t)=t^{-n / \lambda}\left(C-\frac{p-2}{p} \lambda^{1 /(1-p)}\left(\frac{|x|}{t^{1 / \lambda}}\right)^{p /(p-1)}\right)_{+}^{(p-1) /(p-2)} .
$$


Here $p>2$ and $\lambda=n(p-2)+p$. Another example is the equation

$$
\frac{\partial u}{\partial t}=\sum_{i=1}^{n} \frac{\partial}{\partial x_{i}}\left(\left|\frac{\partial u}{\partial x_{i}}\right|^{p-2} \frac{\partial u}{\partial x_{i}}\right)
$$

which was introduced by Lions in [14]. Observe that the equation is separable in the stationary case. In $\Xi=\mathbb{R}^{n} \times(0, \infty)$, we have the weak solution

$$
u(x, t)=t^{-n / \lambda}\left(\frac{p-2}{p} \lambda^{\frac{1}{p-1}}\left(C-\sum_{i=1}^{n}\left(\frac{\left|x_{i}\right|}{t^{1 / \lambda}}\right)^{p /(p-1)}\right)\right)_{+}^{(p-1) /(p-2)},
$$

where $\lambda=n(p-2)+p$ and $C>0$. We extend the solution above to the lower half-space as follows

$$
\mathcal{L}(x, t)=\left\{\begin{aligned}
u(x, t), & (x, t) \in \mathbb{R}^{n} \times(0, \infty), \\
0, & \text { otherwise. }
\end{aligned}\right.
$$

The function $\mathcal{L}$ is a classical solution to equation $(2.5)$ in the open set $\left(\mathbb{R}^{n} \times\right.$ $\mathbb{R}) \backslash \partial\{\mathcal{L}>0\}$. The exceptional set has a measure zero. Despite this fact, the extended function $\mathcal{L}$ is not a weak solution or even a weak supersolution, because the summability of the gradient fails. Nevertheless, the function $\mathcal{L}$ belongs to a broader class of $\mathcal{A}$-superparabolic functions:

Definition 2.6. Let $\Xi$ be an open set in $\mathbb{R}^{n} \times \mathbb{R}$. A function $u: \Xi \rightarrow$ $(-\infty, \infty]$ is called $\mathcal{A}$-superparabolic if

(i) $u$ is lower semicontinuous,

(ii) $u$ is finite in a dense subset of $\Xi$,

(iii) $u$ satisfies the comparison principle on each space-time box $Q_{t_{1}, t_{2}} \Subset$ $\Xi$ : If $h$ is $\mathcal{A}$-parabolic in $Q_{t_{1}, t_{2}}$ and continuous on $\bar{Q}_{t_{1}, t_{2}}$, and, if $h \leq u$ on $\partial_{p} Q_{t_{1}, t_{2}}$, then $h \leq u$ in the whole $Q_{t_{1}, t_{2}}$.

We later prove that $\mathcal{A}$-superparabolic functions satisfy stronger comparison principles. More precisely, they satisfy comparison in all space-time cylinders $U_{t_{1}, t_{2}}$ when using the parabolic boundary, as well as in all open sets $\Xi \subset \mathbb{R}^{n} \times \mathbb{R}$ when using the Euclidean boundary.

There is a well-recognized difficulty in proving estimates for weak solutions. One often needs a test function that depends on $u$ itself, but $u$ does not belong to the class of admissible test functions. For instance, one can easily show that the step function

$$
u(x, t)= \begin{cases}0, & t \leq 0 \\ 1, & t>0\end{cases}
$$

$x \in \mathbb{R}^{n}$, is a weak supersolution but its time derivative is only a generalized function. Nonetheless, there are various techniques to treat this difficulty. One possible way is to use the standard mollification to regularize the solution in the time direction. We set

$$
\phi_{h}(x, t)=\int_{\mathbb{R}} \phi(x, t-s) \rho(h, s) d s,
$$

where $\phi \in C_{0}^{\infty}\left(U \times\left(t_{1}, t_{2}\right)\right)$, for simplicity, and $\rho(h, \cdot)$ stands for a standard mollifier, whose support is contained in $(-h, h), h<\operatorname{dist}(\operatorname{spt}(\phi), U \times$ 
$\left.\left\{t_{1}, t_{2}\right\}\right)$. We insert $\phi_{h}(x, t)$ into (2.4), change variables, and apply Fubini's theorem. As a result, we obtain for supersolutions (subsolutions) the regularized integral inequality, which reads as

$$
-\int_{t_{1}}^{t_{2}} \int_{U} u_{h} \frac{\partial \phi}{\partial t} d x d t+\int_{t_{1}}^{t_{2}} \int_{U} \mathcal{A}(x, t, \nabla u)_{h} \cdot \nabla \phi d x d t \geq(\leq) 0,
$$

where $(\cdot)_{h}$ denotes the mollified function in the time direction.

We use the following existence result for the obstacle problem, see, for instance, Alt-Luckhaus [1] and Korte-Kuusi-Siljander [12].

Theorem 2.8. Let $U$ be a finite union of boxes in $\mathbb{R}^{n}$. Let $\psi \in C^{\infty}\left(U_{t_{1}, t_{2}}\right) \cap$ $C\left(\bar{U}_{t_{1}, t_{2}}\right)$. Then there exists a weak supersolution $u \in C\left(\bar{U}_{t_{1}, t_{2}}\right)$ such that

$$
\begin{aligned}
& u \geq \psi \text { in } K, \\
& u=\psi \text { on } \partial_{p} K, \text { and } \\
& u \text { is } \mathcal{A} \text {-parabolic in }\{u>\psi\} .
\end{aligned}
$$

Moreover, we use the existence of continuous solutions in the space-time boxes with continuous boundary values on the parabolic boundary, see Lions [14], Showalter [15], and DiBenedetto [4].

Theorem 2.9. Let $U$ be a finite union of boxes in $\mathbb{R}^{n}$ and suppose that $\psi \in C\left(\partial_{p} U_{t_{1}, t_{2}}\right)$. Then there exists an $\mathcal{A}$-parabolic function $u \in C\left(\bar{U}_{t_{1}, t_{2}}\right)$ attaining continuously the boundary values

$$
u=\psi \quad \text { on } \partial_{p} U_{t_{1}, t_{2}} .
$$

\section{Parabolic modifications}

Technical difficulties in studying weak supersolutions and $\mathcal{A}$-superparabolic functions sometimes emerge in different situations. For example, the following proposition is a direct consequence of the definition of $\mathcal{A}$-superparabolic functions via comparison.

Proposition 3.1. Let $\Xi$ be an open set in $\mathbb{R}^{n} \times \mathbb{R}$. Suppose that $u$ and $v$ are $\mathcal{A}$-superparabolic (A-subparabolic) in $\Xi$. Then also $\min \{u, v\}$ is $\mathcal{A}$ superparabolic ( $\max \{u, v\}$ is $\mathcal{A}$-subparabolic).

The corresponding result holds also for weak supersolutions. The proof, however, requires more work.

Lemma 3.2. Let $\Xi$ be an open set in $\mathbb{R}^{n} \times \mathbb{R}$. Suppose that $u$ and $v$ are weak supersolutions (subsolutions) to equation (1.1) in $\Xi$. Then also $\min \{u, v\}$ is a weak supersolution $(\max \{u, v\}$ is a subsolution).

Proof. Let then $\varphi \in C_{0}^{\infty}\left(U_{t_{1}, t_{2}}\right), U_{t_{1}, t_{2}} \Subset \Xi$, be non-negative and $0<\varepsilon<1$. We formally choose test functions $\varphi^{u}=\varphi\left(1-a_{\epsilon}\right)$ and $\varphi^{v}=\varphi a_{\epsilon}$, where

$$
a_{\varepsilon}= \begin{cases}0, & u \leq v \\ \frac{u-v}{\varepsilon}, & v<u<v+\varepsilon \\ 1, & u \geq v+\varepsilon\end{cases}
$$

We insert the test functions into (2.4), sum up the integrals and have 


$$
\begin{aligned}
0 \leq & \int_{\Xi}\left(-u \frac{\partial \varphi^{u}}{\partial t}+\mathcal{A}(x, t, \nabla u) \cdot \nabla \varphi^{u}\right) d x d t \\
& +\int_{\Xi}\left(-v \frac{\partial \varphi^{v}}{\partial t}+\mathcal{A}(x, t, \nabla v) \cdot \nabla \varphi^{v}\right) d x d t .
\end{aligned}
$$

Consequently,

$$
\begin{aligned}
0 \leq & \int_{\Xi}\left(-u \frac{\partial \varphi}{\partial t}\left(1-a_{\varepsilon}\right)-v \frac{\partial \varphi}{\partial t} a_{\varepsilon}\right) d x d t+\int_{\Xi}(u-v) \varphi \frac{\partial a_{\varepsilon}}{\partial t} d x d t \\
& +\int_{\Xi}\left(\mathcal{A}(x, t, \nabla u) \cdot(\nabla \varphi)\left(1-a_{\varepsilon}\right)+\mathcal{A}(x, t, \nabla v) \cdot(\nabla \varphi) a_{\varepsilon}\right) d x d t \\
& +\int_{\Xi}(\mathcal{A}(x, t, \nabla v)-\mathcal{A}(x, t, \nabla u)) \cdot\left(\nabla a_{\varepsilon}\right) \varphi d x d t .
\end{aligned}
$$

The last term above is non-positive because of the monotonicity (2.2) of the operator $\mathcal{A}$. We integrate by parts and obtain

$$
\begin{aligned}
\int_{\Xi}(u-v) \varphi \frac{\partial a_{\varepsilon}}{\partial t} d x d t & =\frac{1}{\varepsilon} \int_{\Xi}(u-v) \varphi \frac{\partial(u-v)}{\partial t} \chi_{\{v<u<v+\varepsilon\}} d x d t \\
& =\frac{1}{2 \varepsilon} \int_{\Xi} \varphi \frac{\partial}{\partial t} b^{2} d x d t \\
& =\frac{-1}{2 \varepsilon} \int_{\Xi} b^{2} \frac{\partial \varphi}{\partial t} d x d t,
\end{aligned}
$$

where

$$
b= \begin{cases}0, & u \leq v \\ u-v, & v<u<v+\varepsilon, \\ \varepsilon, & u \geq v+\varepsilon .\end{cases}
$$

This integral converges to zero as $\varepsilon \rightarrow 0$ since $b^{2} \leq \varepsilon^{2}$. Thus, by the results above,

$$
\begin{aligned}
0 \leq & \liminf _{\varepsilon \rightarrow 0}\left[\int_{\Xi}\left(-u \frac{\partial \varphi}{\partial t}\left(1-a_{\varepsilon}\right)-v \frac{\partial \varphi}{\partial t} a_{\varepsilon}\right) d x d t\right. \\
& \left.+\int_{\Xi}\left(\mathcal{A}(x, t, \nabla u) \cdot(\nabla \varphi)\left(1-a_{\varepsilon}\right)+\mathcal{A}(x, t, \nabla v) \cdot(\nabla \varphi) a_{\varepsilon}\right) d x d t\right] \\
= & -\int_{\Xi} \min \{u, v\} \frac{\partial \varphi}{\partial t} d x d t+\int_{\Xi} \mathcal{A}(x, t, \nabla \min \{u, v\}) \cdot \nabla \varphi d x d t .
\end{aligned}
$$

Since $\varphi$ is arbitrary, we conclude that $\min \{u, v\}$ is a weak supersolution.

To justify the word "formally" above, we reason as follows: We insert the regularized test functions $\varphi_{h}^{u}=\varphi\left(1-\left(a_{\epsilon}\right)_{h}\right)$ and $\varphi_{h}^{v}=\varphi\left(a_{\epsilon}\right)_{h}$ into the mollified integral inequality (2.7) and obtain

$$
\begin{aligned}
0 \leq & \int_{\Xi}\left(-u_{h} \frac{\partial \varphi_{h}^{u}}{\partial t}+\mathcal{A}(x, t, \nabla u)_{h} \cdot \nabla \varphi_{h}^{u}\right) d x d t \\
& +\int_{\Xi}\left(-v_{h} \frac{\partial \varphi_{h}^{v}}{\partial t}+\mathcal{A}(x, t, \nabla v)_{h} \cdot \nabla \varphi_{h}^{v}\right) d x d t
\end{aligned}
$$

The test functions are admissible (due to a similar approximation in $\mathbb{R}^{n}$ ) and, in particular, smooth in the time direction. We obtain (3.3) with mollifications, and then integrate by parts as in (3.4) and get rid of the time 
derivatives. Since the resulting equation does not contain time derivatives, we can pass to limit with respect to $h$ by using the properties of mollifiers. As a next step, we again use monotonicity and conclude the proof as above (see also cf. the proof of Theorem 5.3).

Next we state the comparison principle for weak super- and subsolutions. The proof requires similar methods as Lemma 3.2, as shown by Kilpeläinen and Lindqvist in Lemma 3.1 of [9]. For $\mathcal{A}$-super- and $\mathcal{A}$-subparabolic functions, the result follows immediately from the definitions.

Lemma 3.5. Suppose that $u$ is a weak supersolution and $v$ is a weak subsolution to equation (1.1) in a space-time cylinder $U_{t_{1}, t_{2}}$, where $U \subset \mathbb{R}^{n}$ is an open set. If $u$ and $-v$ are lower semicontinuous on $\bar{U}_{t_{1}, t_{2}}$ and $v \leq u$ on the parabolic boundary $\partial_{p} U_{t_{1}, t_{2}}$, then $v \leq u$ almost everywhere in $U_{t_{1}, t_{2}}$.

Proof. We test formally equations of $u$ and $v$ with $\varphi=(v-u-\varepsilon)_{+} \eta, \varepsilon>0$, where $\eta$ is a decreasing Lipschitz function depending only on $t$ such that $\eta=t_{2}-\varepsilon-t$ for $t<t_{2}-\varepsilon$ and $\eta=0$ for $t \geq t_{2}-\varepsilon$. Due to the upper semicontinuity of $v-u$ and the condition $v \leq u$ on the parabolic boundary, the test function $\varphi$ is compactly supported in $U_{t_{1}, t_{2}}$. Thus we may regularize it as in the above discussion in such way that the regularized test function remains compactly supported in $U_{t_{1}, t_{2}}$ for all small enough $h$. Indeed, this justifies the formal calculations below, provided that the final estimate does not contain the "forbidden" time derivative of $\varphi$. This we guarantee by integration by parts.

We first subtract the equations for $u$ and $v$ and obtain

$$
0 \geq \int_{U_{t_{1}, t_{2}}}\left(-(v-u) \frac{\partial \varphi}{\partial t}+(\mathcal{A}(x, t, \nabla v)-\mathcal{A}(x, t, \nabla u)) \cdot \nabla \varphi\right) d x d t .
$$

The monotonicity condition (2.2) gives

$$
(\mathcal{A}(x, t, \nabla v)-\mathcal{A}(x, t, \nabla u)) \cdot \nabla(v-u-\varepsilon)_{+} \geq 0
$$

for almost every $(x, t)$ in $U_{t_{1}, t_{2}}$ implying

$$
0 \leq \int_{U_{t_{1}, t_{2}}}(v-u) \frac{\partial \varphi}{\partial t} d x d t
$$

We integrate by parts and obtain

$$
\begin{aligned}
\int_{U_{t_{1}, t_{2}}}(v-u) \frac{\partial \varphi}{\partial t} d x d t & =\frac{1}{2} \int_{U_{t_{1}, t_{2}}}(v-u-\varepsilon)_{+}^{2} \frac{\partial \eta}{\partial t} d x d t \\
& =-\frac{1}{2} \int_{U_{t_{1}, t_{2}-\varepsilon}}(v-u-\varepsilon)_{+}^{2} d x d t .
\end{aligned}
$$

This leads to

showing that

$$
0 \geq \frac{1}{2} \int_{U_{t_{1}, t_{2}-\varepsilon}}(v-u-\varepsilon)_{+}^{2} d x d t
$$

$$
v \leq u+\varepsilon \text { almost everywhere in } U_{t_{1}, t_{2}-\varepsilon} .
$$

This holds for all $\varepsilon>0$ proving the result. 
We use the comparison principle to prove that a weak subsolution can be modified by an $\mathcal{A}$-parabolic function in a space-time cylinder so that it remains a weak subsolution.

Lemma 3.6. Let $\Omega_{T}=\Omega \times(0, T)$ and $U_{t, T}=U \times(t, T)$ be open sets in $\mathbb{R}^{n} \times \mathbb{R}$ such that $U_{t, T} \subset \Omega_{T}$. Let $u$ be a continuous weak subsolution in $\Omega_{T}$ and $h$ be an $\mathcal{A}$-parabolic function in $U_{t, T}$. Suppose that $u$ and $h$ are continuous up to the parabolic boundaries of $\Omega_{T}$ and $U_{t, T}$, respectively, and that $u=h$ on $\partial_{p} U_{t, T}$. Then

$$
w= \begin{cases}h & \text { in } U_{t, T} \\ u & \text { in } \Omega_{T} \backslash U_{t, T}\end{cases}
$$

is a continuous subsolution in $\Omega_{T}$.

Proof. By Lemma 3.5, $h \geq u$ in $U_{t, T}$. Let $\varphi \in C_{0}^{\infty}\left(\Omega_{T}\right)$ be non-negative and $0<\varepsilon<1$. We formally choose test functions $\varphi^{h}=\varphi a_{\varepsilon}$ and $\varphi^{u}=\varphi\left(1-a_{\varepsilon}\right)$, where $a_{\varepsilon}=0$ in $\Omega_{T} \backslash U_{t, T}$ and $a_{\varepsilon}$ is defined in $U_{t, T}$ as

$$
a_{\varepsilon}= \begin{cases}0, & h \leq u+\varepsilon, \\ \frac{h-u-\varepsilon}{\varepsilon}, & u+\varepsilon<h<u+2 \varepsilon, \\ 1, & h \geq u+2 \varepsilon .\end{cases}
$$

Now $\varphi^{h}$ has compact support in $U_{t, T}$. Then

$$
\begin{aligned}
0 \geq & \int_{\Omega_{T}}\left(-h \frac{\partial \varphi^{h}}{\partial t}+\mathcal{A}(x, t, \nabla h) \cdot \nabla \varphi^{h}\right) d x d t \\
& +\int_{\Omega_{T}}\left(-u \frac{\partial \varphi^{u}}{\partial t}+\mathcal{A}(x, t, \nabla u) \cdot \nabla \varphi^{u}\right) d x d t,
\end{aligned}
$$

and, consequently,

$$
\begin{aligned}
0 \geq & \int_{\Omega_{T}}\left(-h \frac{\partial \varphi}{\partial t} a_{\varepsilon}-u \frac{\partial \varphi}{\partial t}\left(1-a_{\varepsilon}\right)\right) d x d t \\
& +\int_{\Omega_{T}}\left(\mathcal{A}(x, t, \nabla h) \cdot(\nabla \varphi) a_{\varepsilon}+\mathcal{A}(x, t, \nabla u) \cdot(\nabla \varphi)\left(1-a_{\varepsilon}\right)\right) d x d t \\
& +\int_{\Omega_{T}}(u-h) \varphi \frac{\partial a_{\varepsilon}}{\partial t} d x d t \\
& +\int_{\Omega_{T}}(\mathcal{A}(x, t, \nabla h)-\mathcal{A}(x, t, \nabla u)) \cdot \frac{\nabla(h-u)}{\varepsilon} \chi_{\{u+\varepsilon \leq h \leq u+2 \varepsilon\}} \varphi d x d t .
\end{aligned}
$$

It follows from the monotonicity that

$$
\begin{aligned}
0 \geq & \int_{\Omega_{T}}-h \frac{\partial \varphi}{\partial t} a_{\varepsilon}-u \frac{\partial \varphi}{\partial t}\left(1-a_{\varepsilon}\right) d x d t \\
& +\int_{\Omega_{T}}\left(\mathcal{A}(x, t, \nabla h) \cdot(\nabla \varphi) a_{\varepsilon}+\mathcal{A}(x, t, \nabla u) \cdot(\nabla \varphi)\left(1-a_{\varepsilon}\right)\right) d x d t \\
& +\int_{\Omega_{T}}(u-h) \varphi \frac{\partial a_{\varepsilon}}{\partial t} d x d t .
\end{aligned}
$$


Integrating by parts, we get

$$
\begin{aligned}
\int_{\Omega_{T}}(u-h) \varphi \frac{\partial a_{\varepsilon}}{\partial t} d x d t & =-\int_{\Omega_{T}} \frac{1}{\varepsilon}(u-h) \varphi \frac{\partial(u-h)}{\partial t} \chi_{\{u+\varepsilon \leq h \leq u+2 \varepsilon\}} d x d t \\
& =\frac{-1}{2 \varepsilon} \int_{\Omega_{T}} \varphi \frac{\partial}{\partial t} b^{2} d x d t \\
& =\frac{1}{2 \varepsilon} \int_{\Omega_{T}} b^{2} \frac{\partial \varphi}{\partial t} d x d t
\end{aligned}
$$

where

$$
b= \begin{cases}\varepsilon, & h<u+\varepsilon \\ h-u, & u+\varepsilon \leq h \leq u+2 \varepsilon \\ 2 \varepsilon, & h \geq u+2 \varepsilon\end{cases}
$$

This integral converges to zero as $\varepsilon \rightarrow 0$ since $b^{2} \leq 4 \varepsilon^{2}$. Hence

$$
\begin{aligned}
0 \geq & \limsup _{\varepsilon \rightarrow 0}\left[\int_{\Omega_{T}}\left(-h \frac{\partial \varphi^{h}}{\partial t}+\mathcal{A}(x, t, \nabla h) \cdot \nabla \varphi^{h}\right) d x d t\right. \\
& \left.+\int_{\Omega_{T}}\left(-u \frac{\partial \varphi^{u}}{\partial t}+\mathcal{A}(x, t, \nabla u) \cdot \nabla \varphi^{u}\right) d x d t\right] \\
\geq & -\int_{\Omega_{T}} w \frac{\partial \varphi}{\partial t} d x d t+\int_{\Omega_{T}} \mathcal{A}(x, t, \nabla w) \cdot \nabla \varphi d x d t
\end{aligned}
$$

and $w$ is a continuous subsolution.

We employ Lemma 3.6 in this extent to prove more general comparison results in Section 4. However, after proving the comparison results, we see that cylinders can be replaced by more general sets in the statement of Lemma 3.6.

\section{Comparison of $\mathcal{A}$-superparabolic functions}

In the definition of $\mathcal{A}$-superparabolic functions, we a priori assume that they satisfy the comparison principle in space-time boxes. Nonetheless, $\mathcal{A}$ superparabolic functions also satisfy stronger comparison principles: First, we show that the comparison principle holds in the union of a finite number of space-time boxes having the same time interval. This step requires most of the work. Next we extend the result to the union of a finite number of space-time boxes with varying time intervals. Furthermore, the result extends to any open set in $\mathbb{R}^{n+1}$ if we compare the boundary values on the whole Euclidean boundary. In particular, the comparison principle holds in any open cylinder $U_{t_{1}, t_{2}}$, and, thus, we can replace space-time boxes with open cylinders in the condition (iii) of Definition 2.6.

The next lemma deals with a finite union of space-time boxes having the same time interval. We employ a parabolic version of the Schwarz alternating method.

Lemma 4.1. Let $Q_{i} \subset \mathbb{R}^{n}$ be a box for $i=1,2, \ldots, N$ and let $Q_{t_{1}, t_{2}}^{i}=$ $Q_{i} \times\left(t_{1}, t_{2}\right)$. Let $K=\cup_{i=1}^{N} Q_{t_{1}, t_{2}}^{i}$ and let $u$ be $\mathcal{A}$-superparabolic in the neighborhood of $\bar{K}$. If $h$ is $\mathcal{A}$-parabolic in $K$ and continuous in $\bar{K}$, and, if $h \leq u$ on $\partial_{p} K$, then $h \leq u$ in the whole $K$. 
Proof. Let $\delta>0$ and choose a function $\varphi \in C^{\infty}(K) \cap C(\bar{K})$ such that $\varphi \leq u$ in $K \cup \partial_{p} K$ and $h-\delta \leq \varphi$ on $\partial_{p} K$. By Theorem 2.8, there exists a continuous subsolution $\psi_{0}$, which belongs to

$$
\left\{\psi \in C(\bar{K}): \psi=\varphi \text { on } \partial_{p} K \text { and } \psi \leq \varphi \text { in } K\right\} .
$$

Starting from $\psi_{0}$, we construct an increasing sequence of subsolutions $v_{k}, k=0,1,2, \ldots$, converging to an $\mathcal{A}$-parabolic function. Set $v_{0}=\psi_{0}$. For all $j \geq 0,1 \leq i \leq N$, let $v_{N j+i}$ be $\mathcal{A}$-parabolic in $Q_{t_{1}, t_{2}}^{i}$ with boundary values $v_{N j+i-1}$ and $v_{N j+i}=v_{N j+i-1}$ in $K \backslash Q_{t_{1}, t_{2}}^{i}$.

Each $v_{k}, k=1,2, \ldots$, is a continuous subsolution in $K$ by Lemma 3.6. Moreover, the sequence $v_{k}$ is increasing by the comparison principle for subsolutions, and $v_{N j+i} \leq u$ in $\bar{K}$ by the comparison principle for supersolutions. Indeed, $v_{N j+i}$ is $\mathcal{A}$-parabolic in $Q_{t_{1}, t_{2}}^{i}$, and, hence, we can employ the comparison principles in $Q_{t_{1}, t_{2}}^{i}$ between $v_{N j+i}$ and $v_{N j+i-1}$, as well as between $v_{N j+i}$ and $u$. Consequently, the limit $w=\lim _{k \rightarrow \infty} v_{k}$ exists and

$$
w \leq u \text { in } \bar{K} .
$$

Next we show that $w$ is continuous in $K$. To this end, fix $z \in K$ and $i_{z}$ such that $z \in Q_{t_{1}, t_{2}}^{i_{z}}$. By construction, $v_{N j+i_{z}}, j=0,1, \ldots$, are $\mathcal{A}$-parabolic in $Q_{t_{1}, t_{2}}^{i_{z}}$. Hence, they are Hölder continuous in $B\left(z, r_{z}\right) \subset Q_{t_{1}, t_{2}}^{i_{z}}$, for some $r_{z}>0$, with constants independent of $j$, see DiBenedetto [4] or DiBenedettoUrbano-Vespri [6]. It follows that the monotonic subsequence $v_{N j+i_{z}}, j=$ $0,1, \ldots$, converges to a continuous function, which must be $w$. Hence $w$ is continuous in $K$.

The limit $w$ is also continuous up to the boundary. Indeed, let $h_{\varphi}$ be an $\mathcal{A}$-parabolic function in $K$ with the boundary values $\varphi$ on $\partial_{p} K$. Collecting the facts, we have

$$
\begin{aligned}
& v_{0} \leq w \leq h_{\varphi} \text { in } K \cup \partial_{p} K, \\
& v_{0}=h_{\varphi} \text { on } \partial_{p} K, \text { and } \\
& v_{0} \text { and } h_{\varphi} \text { are continuous in } K \cup \partial_{p} K .
\end{aligned}
$$

Hence $w$ is continuous in $K \cup \partial_{p} K$.

Finally, we prove that $w$ is $\mathcal{A}$-parabolic in $K$. It suffices to prove that for each $1 \leq i_{0} \leq N$ and $\rho>0, w$ is a solution in $Q_{t_{1}, t_{2}-\rho}^{i_{0}}$. First, because $v_{k}$, $k=1,2, \ldots$, and $w$ are continuous, it follows that the functions $v_{k}$ converge uniformly to $w$ in $\bar{K} \cap\{t \leq T-\rho\}$ for every $\rho>0$ as $k \rightarrow \infty$. Thus, for every $\varepsilon>0$, there exists $j_{\varepsilon}$ such that

$$
\left|w-v_{N j_{\varepsilon}+i_{0}}\right|<\varepsilon \quad \text { on } \quad \bar{Q}_{t_{1}, t_{2}-\rho}^{i_{0}} .
$$

To continue, let $w^{\prime}$ be an $\mathcal{A}$-parabolic function in $Q_{t_{1}, t_{2}-\rho}^{i_{0}}$ with boundary values $w$ on $\partial_{p} Q_{t_{1}, t_{2}-\rho}^{i_{0}}$. By (4.3), we have

$$
v_{N j_{\varepsilon}+i_{0}}-\varepsilon \leq w^{\prime} \leq v_{N j_{\varepsilon}+i_{0}}+\varepsilon \quad \text { on } \quad \partial_{p} Q_{t_{1}, t_{2}-\rho}^{i_{0}}
$$

and, because $v_{N j_{\varepsilon}+i_{0}}$ and $w^{\prime}$ are solutions in $Q_{t_{1}, t_{2}-\rho}^{i_{0}}$, the comparison principle implies

$$
v_{N j_{\varepsilon}+i_{0}}-\varepsilon \leq w^{\prime} \leq v_{N j_{\varepsilon}+i_{0}}+\varepsilon \quad \text { on } \quad \bar{Q}_{t_{1}, t_{2}-\rho}^{i_{0}} .
$$


Hence

$$
\left|w-w^{\prime}\right| \leq\left|w-v_{N j_{\varepsilon}+i_{0}}\right|+\left|v_{N j_{\varepsilon}+i_{0}}-w^{\prime}\right|<2 \varepsilon \quad \text { on } \quad \bar{Q}_{t_{1}, t_{2}-\rho}^{i_{0}} .
$$

Because $\varepsilon$ was arbitrary, it follows that $w^{\prime}=w$ in $Q_{t_{1}, t_{2}-\rho}^{i_{0}}$ and, consequently, $w$ is a solution in $Q_{t_{1}, t_{2}-\rho}^{i_{0}}$. Thus $w$ is $\mathcal{A}$-parabolic in $K$, and by the comparison principle for $\mathcal{A}$-parabolic functions, $h-\delta \leq w$ in $K$. Letting $\delta \rightarrow 0$ provides the inequality $h \leq w$ in $K$, and, on the other hand, (4.2) implies that $w \leq u$ in $\bar{K}$. This proves the claim.

Next we consider the comparison principle in the union of finite number of space-time boxes with different time intervals.

Lemma 4.4. Let $Q_{i} \subset \mathbb{R}^{n}$ be a box for $i=1,2, \ldots, N$, let $Q_{t_{1}^{i}, t_{2}^{i}}^{i}=Q_{i} \times$ $\left(t_{1}^{i}, t_{2}^{i}\right)$, and let $K=\cup_{i=1}^{N} Q_{t_{1}^{i}, t_{2}^{i}}^{i}$. Furthermore, let $u$ be $\mathcal{A}$-superparabolic in the neighborhood of $\bar{K}$. If $h$ is $\mathcal{A}$-parabolic in $K$ and continuous on $\bar{K}$ and if $h \leq u$ on $\partial_{p} K$, then $h \leq u$ in $K$.

Proof. Let $s_{1}<s_{2}<\ldots<s_{M}$, be the set of points $t_{j}^{i}, i=1,2, \ldots, N$, $j=1,2$, in an increasing order. By Lemma 4.1, it follows that $h \leq u$ in $K \cap\left\{t \leq s_{2}\right\}$. By induction, the same result holds for all $K \cap\left\{s_{i} \leq t \leq\right.$ $\left.s_{i+1}\right\}$.

In the following lemmas, we extend the $\mathcal{A}$-superparabolic functions up to the boundary as

$$
u(x, t)=\liminf _{(y, s) \rightarrow(x, t)} u(y, s), \quad(x, t) \in \partial \Xi .
$$

If we consider the whole Euclidean boundary, then the comparison principle holds in every open bounded set.

Lemma 4.5. Let $\Xi$ be an open bounded set in $\mathbb{R}^{n} \times \mathbb{R}$, let $u$ be $\mathcal{A}$ superparabolic in $\Xi$. Let $h$ be $\mathcal{A}$-parabolic in $\Xi$ and continuous on $\Xi$. If $h \leq u$ on $\partial \Xi$, then $h \leq u$ in the whole $\Xi$.

Proof. Let $\varepsilon>0$ and denote

$$
D=\{(x, t) \in \bar{\Xi}: h(x, t) \geq u(x, t)+\varepsilon\} .
$$

The set $D$ is compact and due to the boundary condition $D \subset \Xi$. Consequently, there exists a finite union of space-time boxes $Q_{t_{1}^{i}, t_{2}^{i}}^{i}$ with closures in $\Xi$ such that $D \subset K=\cup Q_{t_{1}^{i}, t_{2}^{i}}^{i}$. Because $\partial_{p} K \subset \Xi \backslash D$, we have $h<u+\varepsilon$ on $\partial_{p} K$. Thus, by Lemma 4.4, we obtain $h \leq u+\varepsilon$ in $K$. Because $D \subset K$, it follows that $h \leq u+\varepsilon$ in $\Xi$ and the result follows.

The following corollary shows that the comparison principle holds in a set $\Xi \cap\{t<T\}$.

Corollary 4.6. Let $\Xi$ be an open set in $\mathbb{R}^{n} \times \mathbb{R}$. Let $\Xi_{T}=\Xi \cap\{t<T\}$ and

$$
\partial_{T} \Xi_{T}=\partial \Xi \cap\{t<T\} .
$$

Let $u$ be $\mathcal{A}$-superparabolic in $\Xi_{T}$, let $h$ be $\mathcal{A}$-parabolic in $\Xi_{T}$, continuous on $\bar{\Xi}_{T}$, and let $h \leq u$ on $\partial_{T} \Xi_{T}$. Then $h \leq u$ in $\Xi_{T}$. 
Proof. Fix $\varepsilon>0$ and let

$$
D_{T-\varepsilon}=\left\{(x, t) \in \bar{\Xi}_{T-\varepsilon}: h(x, t) \geq u(x, t)+\varepsilon\right\} .
$$

Similarly as in Lemma 4.5 , we conclude that $D_{T-\varepsilon}$ is compact and $D_{T-\varepsilon} \subset$ $\Xi_{T}$. Hence, there exists a finite set of space-time boxes $Q_{t_{1}^{i}, t_{2}^{i}}^{i}$ with closures in $\Xi_{T}$ such that $D_{T-\varepsilon} \subset K=\cup Q_{t_{1}^{i}, t_{2}^{i}}^{i}$ and $\partial_{p}(K \cap\{t<T-\varepsilon\}) \subset \Xi_{T} \backslash D_{T}$. The result then follows as in the proof of Lemma 4.5.

By setting $\Xi=U \times\left(t_{1}, t_{2}\right)$, where $U$ is open, we obtain the following corollary.

Corollary 4.7. The condition (iii) in the Definition 2.6 of $\mathcal{A}$-superparabolic functions can be replaced by an equivalent condition

(iii') $u$ satisfies the comparison principle on each cylinder $U_{t_{1}, t_{2}}=U \times$ $\left(t_{1}, t_{2}\right) \Subset \Xi$, $U$ open in $\mathbb{R}^{n}$ : If $h$ is $\mathcal{A}$-parabolic in $U_{t_{1}, t_{2}}$ and continuous on $\bar{U}_{t_{1}, t_{2}}$, and, if $h \leq u$ on $\partial_{p} U_{t_{1}, t_{2}}$, then $h \leq u$ in the whole $U_{t_{1}, t_{2}}$.

In particular, we end up with the same class of $\mathcal{A}$-superparabolic functions if we require that they satisfy the comparison in more general sets than space-time boxes. This property seems to be useful in the applications. The condition (iii') has been used in the definition of the superparabolic functions, for example, in [11] by Kinnunen and Lindqvist.

The following version of the comparison principle might be interesting in its own right although we do not use it here. After establishing Lemma 4.4 and further showing that in Theorem 2.8 the set $U_{t_{1}, t_{2}}$ may be replaced with a finite union of space-time cylinders by a similar argument as in the proof of Lemma 4.4, the proof of the following theorem is the same as of Lemma 4.3 in Kilpeläinen-Lindqvist [9].

Theorem 4.8. Let $\Xi$ be an open bounded set in $\mathbb{R}^{n} \times \mathbb{R}$. Suppose that $u$ is $\mathcal{A}$-superparabolic and $v$ is $\mathcal{A}$-subparabolic in $\Xi$. If

$$
\limsup _{(y, s) \rightarrow(x, t)} v(y, s) \leq \liminf _{(y, s) \rightarrow(x, t)} u(y, s)
$$

for all $(x, t) \in \partial \Xi$ and both sides are not simultaneously $\infty$ or $-\infty$, then $v \leq u$ in $\Xi$.

\section{Convergence of supersolutions}

In this section, we prove that a bounded $\mathcal{A}$-superparabolic function is a weak supersolution. To accomplish this, we first show that a pointwise limit of a sequence of uniformly bounded weak supersolutions is a weak supersolution.

To begin with, observe that $\mathcal{A}$-superparabolic functions form a closed class with respect to the upper directed convergence.

Proposition 5.1. Let $u_{i}, i=1,2, \ldots$, be an increasing sequence of $\mathcal{A}$-superparabolic functions in an open set $\Xi$ in $\mathbb{R}^{n} \times \mathbb{R}$. Suppose that $u=\sup u_{i}$ is finite in a dense subset of $\Xi$. Then $u$ is $\mathcal{A}$-superparabolic. 
Proof. The function $u$, as the supremum of lower semicontinuous functions, is lower semicontinuous. To show the comparison, we first fix $\varepsilon>0$ and choose $Q_{t_{1}, t_{2}} \Subset \Xi$. To continue, let $h$ be an $\mathcal{A}$-parabolic function in $Q_{t_{1}, t_{2}}$ such that $u \geq h$ on $\partial_{p} Q_{t_{1}, t_{2}}$. By the lower semicontinuity of $u_{i}-h$, the set $\Xi \cap\left\{u_{i}>h-\varepsilon\right\}$ is open. The collection $\left\{\Xi \cap\left\{u_{i}>h-\varepsilon\right\}\right\}$ covers $\partial_{p} Q_{t_{1}, t_{2}}$. The compactness of $\partial_{p} Q_{t_{1}, t_{2}}$ and the monotonicity of the sequence implies that there exists $k$ such that $u_{k} \geq h-\varepsilon$ on $\partial_{p} Q_{t_{1}, t_{2}}$. Since $u_{k}$ is $\mathcal{A}$-superparabolic, it follows that $u_{k} \geq h-\varepsilon$ in $Q_{t_{1}, t_{2}}$, and, thus, $u \geq h-\varepsilon$ in $Q_{t_{1}, t_{2}}$. Since $\varepsilon>0$ is arbitrary, the result follows.

The corresponding result does not hold for supersolutions: A direct calculation shows (see also Lemma 3.2) that the truncated function $\mathcal{L}_{k}=$ $\min (\mathcal{L}, k)$, where $k \in \mathbb{R}$, and $\mathcal{L}$ is as in Section 2 , is a weak supersolution. However, the limit $\mathcal{L}$ of the sequence $\mathcal{L}_{k}, k=1,2, \ldots$, is not a weak supersolution. Nonetheless, any pointwise limit of a sequence of uniformly bounded weak supersolutions is a weak supersolution as we will show in Theorem 5.3.

The following lemma provides a well-known estimate for the gradient of a bounded supersolution. The proof employs the test function $\varphi(x, t)=$ $(M-u(x, t)) \eta^{p}(x, t)$ together with the definition of a weak supersolution.

Lemma 5.2 (Caccioppoli). Let $\Xi$ be an open set in $\mathbb{R}^{n} \times \mathbb{R}$, and let $u$ be a weak supersolution such that $-M \leq u \leq M$. Then there exists a constant $C=C(\alpha, \beta, p)>0$ such that

$$
\int_{\Xi} \eta^{p}|\nabla u|^{p} d x d t \leq C M^{2} \int_{\Xi}\left|\frac{\partial \eta^{p}}{\partial t}\right| d x d t+C M^{p} \int_{\Xi}|\nabla \eta|^{p} d x d t,
$$

whenever $\eta \in C_{0}^{\infty}(\Xi)$, and $\eta \geq 0$.

Next we show that a pointwise limit of a sequence of uniformly bounded weak supersolutions is a weak supersolution following the guidelines of Theorem 6 in Lindqvist and Manfredi [13]. To extend results for more general operators, we employ techniques from Boccardo et. al. [3] to obtain the convergence of gradients in measure. Observe that the proof does not distinguish between increasing and decreasing convergence for supersolutions.

The double limit procedure with respect to the sequence of supersolutions and the regularization parameter might cause subtle difficulties as pointed out by Kinnunen and Lindqvist in Remark 4.1 of [11]. Therefore, we carefully write down the regularizations.

Theorem 5.3. Let $\Xi$ be an open set in $\mathbb{R}^{n} \times \mathbb{R}$. Suppose that $u_{i}$, $i=$ $1,2, \ldots$ is a sequence of supersolutions in $\Xi$ such that $-M \leq u_{i} \leq M$ and $u_{i}$ converges to $u$ almost everywhere in $\Xi$. Then $u$ is a weak supersolution.

Proof. The pointwise convergence implies a convergence in measure in a bounded domain, and thus, the set $\left\{\left|u_{j}-u_{k}\right| \geq \delta\right\}$ will be small for $j$ and $k$ large enough. It remains to derive an integral estimate in a set $\left\{\left|u_{j}-u_{k}\right|<\right.$ $\delta\}$. To this end, first choose a cut-off function $\theta \in C_{0}^{\infty}\left(Q_{\tau_{1}, \tau_{2}}^{\prime}\right)$ such that $\theta=1$ in $Q_{t_{1}, t_{2}}$, where $Q_{t_{1}, t_{2}} \Subset Q_{\tau_{1}, \tau_{2}}^{\prime}=Q^{\prime} \times\left(\tau_{1}, \tau_{2}\right) \Subset \Xi$. Let $h, \delta>0$. Then choose the test functions

$$
\varphi_{1}^{h}=\left(\delta-w_{j k}^{h}\right) \theta
$$


and

where

$$
\varphi_{2}^{h}=\left(\delta+w_{j k}^{h}\right) \theta
$$

$$
w_{j k}^{h}= \begin{cases}\delta, & \left(u_{j}-u_{k}\right)_{h}>\delta \\ \left(u_{j}-u_{k}\right)_{h}, & \left|\left(u_{j}-u_{k}\right)_{h}\right| \leq \delta \\ -\delta, & \left(u_{j}-u_{k}\right)_{h}<-\delta\end{cases}
$$

Observe that $w_{j k}^{h} \in[-\delta, \delta]$ implying $\varphi_{1}^{h}, \varphi_{2}^{h} \geq 0$.

We use (2.7) to have

$$
\int_{Q_{\tau_{1}, \tau_{2}}^{\prime}} \mathcal{A}\left(x, t, \nabla u_{j}\right)_{h} \cdot \nabla \varphi_{1}^{h} d x d t-\int_{Q_{\tau_{1}, \tau_{2}}^{\prime}}\left(u_{j}\right)_{h} \frac{\partial \varphi_{1}^{h}}{\partial t} d x d t \geq 0,
$$

and

$$
\int_{Q_{\tau_{1}, \tau_{2}}^{\prime}} \mathcal{A}\left(x, t, \nabla u_{k}\right)_{h} \cdot \nabla \varphi_{2}^{h} d x d t-\int_{Q_{\tau_{1}, \tau_{2}}^{\prime}}\left(u_{k}\right)_{h} \frac{\partial \varphi_{2}^{h}}{\partial t} d x d t \geq 0 .
$$

To continue, we sum up the inequalities, arrange the terms, and end up with

$$
\begin{aligned}
& \int_{\left\{\left|\left(u_{j}-u_{k}\right)_{h}\right| \leq \delta\right\} \cap Q_{\tau_{1}, \tau_{2}}^{\prime}}\left(\mathcal{A}\left(x, t, \nabla u_{j}\right)-\mathcal{A}\left(x, t, \nabla u_{k}\right)\right)_{h} \cdot \theta \nabla w_{j k}^{h} d x d t \\
& \leq \delta \int_{Q_{\tau_{1}, \tau_{2}}^{\prime}}\left(\mathcal{A}\left(x, t, \nabla u_{j}\right)+\mathcal{A}\left(x, t, \nabla u_{k}\right)\right)_{h} \cdot \nabla \theta d x d t \\
& \quad-\int_{Q_{\tau_{1}, \tau_{2}}^{\prime}}\left(\mathcal{A}\left(x, t, \nabla u_{j}\right)-\mathcal{A}\left(x, t, \nabla u_{k}\right)\right)_{h} w_{j k}^{h} \cdot \nabla \theta d x d t \\
& \quad+\int_{Q_{\tau_{1}, \tau_{2}}^{\prime}}\left(u_{j}-u_{k}\right)_{h} \frac{\partial\left(w_{j k}^{h} \theta\right)}{\partial t} d x d t-\delta \int_{Q_{\tau_{1}, \tau_{2}}^{\prime}}\left(u_{j}+u_{k}\right)_{h} \frac{\partial \theta}{\partial t} d x d t
\end{aligned}
$$

We would like to pass to a limit with respect to $h$, but to accomplish this, the estimate should be free of the time derivatives of $w_{j k}^{h}$. To obtain such an estimate, we integrate by parts and obtain

$$
\begin{aligned}
\int_{Q_{\tau_{1}, \tau_{2}}^{\prime}} & \left(u_{j}-u_{k}\right)_{h} \frac{\partial\left(w_{j k}^{h} \theta\right)}{\partial t} d x d t \\
= & \int_{Q_{\tau_{1}, \tau_{2}}^{\prime}}\left(u_{j}-u_{k}\right)_{h}\left(\frac{\partial w_{j k}^{h}}{\partial t} \theta+w_{j k}^{h} \frac{\partial \theta}{\partial t}\right) d x d t \\
\leq & \frac{1}{2} \int_{\left\{\left|\left(u_{j}-u_{k}\right)_{h}\right| \leq \delta\right\} \cap Q_{\tau_{1}, \tau_{2}}^{\prime}}\left(w_{j k}^{h}\right)^{2} \frac{\partial \theta}{\partial t} d x d t+2 M \int_{Q_{\tau_{1}, \tau_{2}}^{\prime}} \delta\left|\frac{\partial \theta}{\partial t}\right| d x d t \\
\leq & C \delta,
\end{aligned}
$$

where the constant $C$ depends on $\theta$, but not on $j$ and $k$. A similar estimate also holds for the last term (5.4). Furthermore, the properties of mollifiers imply

$$
\begin{aligned}
& \int_{Q_{\tau_{1}, \tau_{2}}^{\prime}} \chi_{\left\{\left|\left(u_{j}-u_{k}\right)_{h}\right| \leq \delta\right\}}\left(\mathcal{A}\left(x, t, \nabla u_{j}\right)-\mathcal{A}\left(x, t, \nabla u_{k}\right)\right)_{h} \cdot \theta \nabla w_{j k}^{h} d x d t \\
& \quad \rightarrow \int_{\left\{\left|u_{j}-u_{k}\right| \leq \delta\right\} \cap Q_{\tau_{1}, \tau_{2}}^{\prime}}\left(\mathcal{A}\left(x, t, \nabla u_{j}\right)-\mathcal{A}\left(x, t, \nabla u_{k}\right)\right) \cdot \theta \nabla\left(u_{j}-u_{k}\right) d x d t,
\end{aligned}
$$


as $h \rightarrow 0$ for almost every $\delta>0$. We also used the fact that $\chi_{\left\{\left|\left(u_{j}-u_{k}\right)_{h}\right| \leq \delta\right\}}$ converges to $\chi_{\left\{\left|u_{j}-u_{k}\right| \leq \delta\right\}}$ in $L^{q}(\Xi)$ for every $1 \leq q<\infty$ and for almost every $\delta>0$. Observe that the values of $\delta$ for which this holds might depend on $j$ and $k$. Nevertheless, we may take a countable intersection of such values of $\delta$. Letting $h \rightarrow 0$ on both sides of (5.4) leads to

$$
\begin{aligned}
& \int_{\left\{\left|u_{j}-u_{k}\right| \leq \delta\right\} \cap Q_{\tau_{1}, \tau_{2}}^{\prime}}\left(\mathcal{A}\left(x, t, \nabla u_{j}\right)-\mathcal{A}\left(x, t, \nabla u_{k}\right)\right) \cdot \theta \nabla\left(u_{j}-u_{k}\right) d x d t \\
& \leq \delta \int_{\left\{\left|u_{j}-u_{k}\right| \leq \delta\right\} \cap Q_{\tau_{1}, \tau_{2}}^{\prime}}\left(\mathcal{A}\left(x, t, \nabla u_{j}\right)+\mathcal{A}\left(x, t, \nabla u_{k}\right)\right) \cdot \nabla \theta d x d t \\
& -\int_{\left\{\left|u_{j}-u_{k}\right| \leq \delta\right\} \cap Q_{\tau_{1}, \tau_{2}}^{\prime}}\left(\mathcal{A}\left(x, t, \nabla u_{j}\right)-\mathcal{A}\left(x, t, \nabla u_{k}\right)\right)\left(u_{j}-u_{k}\right) \cdot \nabla \theta d x d t+C \delta,
\end{aligned}
$$

for almost all $\delta>0$. Next we would like to estimate the terms on the right hand side. To this end, observe that the sequence $\nabla u_{i}, i=1,2, \ldots$ is uniformly bounded in $L^{p}\left(Q_{\tau_{1}, \tau_{2}}^{\prime}\right)$. Indeed, this is a consequence of the fact $-M \leq u_{i} \leq M$ and Caccioppoli's inequality in Lemma 5.2. Therefore, taking into account the growth bounds of $\mathcal{A}$, the first and the second term on the right hand side is bounded by $C \delta$, where $C$ depends on $M$ and $\theta$, but not on $j$ and $k$. Consequently, for almost every $\delta>0$, we have

$$
\int_{\left\{\left|u_{j}-u_{k}\right| \leq \delta\right\}}\left(\mathcal{A}\left(x, t, \nabla u_{j}\right)-\mathcal{A}\left(x, t, \nabla u_{k}\right)\right) \cdot \theta \nabla\left(u_{j}-u_{k}\right) d x d t \leq C \delta
$$

where $C$ does not depend on $j$ and $k$.

Next we recall that the sequence $u_{i}, i=1,2, \ldots$ converges in measure. This implies that for all $\delta, \eta>0$ we can choose large enough $j$ and $k$ so that

$$
\left|\left\{\left|u_{j}-u_{k}\right| \geq \delta\right\}\right|<\eta \text {. }
$$

We also show that the sequence $\nabla u_{i}, i=1,2, \ldots$ converges in measure. Together with the fact that the norms of the gradients are uniformly bounded this implies the pointwise convergence. We define the following sets

$$
\begin{aligned}
& E_{j k}=\left\{(x, t) \in Q_{t_{1}, t_{2}}:\left|\nabla u_{j}(x, t)-\nabla u_{k}(x, t)\right| \geq \rho\right\}, \\
& U_{j k}=\left\{(x, t) \in Q_{t_{1}, t_{2}}:\left|u_{j}(x, t)-u_{k}(x, t)\right| \leq \delta\right\}, \quad \complement U_{j k}=Q_{t_{1}, t_{2}} \backslash U_{j k}, \\
& V_{j k}=\left\{(x, t) \in Q_{t_{1}, t_{2}}:\left|\nabla u_{j}(x, t)\right|,\left|\nabla u_{k}(x, t)\right| \leq \lambda\right\}, \quad \complement V_{j k}=Q_{t_{1}, t_{2}} \backslash V_{j k} .
\end{aligned}
$$

First, we observe that

$$
\left|E_{j k}\right| \leq\left|E_{j k} \cap U_{j k}\right|+\left|C U_{j k}\right|,
$$

and, hence, it is enough to show that the measure of the sets on the right can be made smaller than any $\eta>0$. Observe that since the sequence $u_{i}, i=1,2, \ldots$ converges in measure, it follows that

$$
\left|\complement U_{j k}\right|<\frac{\eta}{3}
$$

for $j$ and $k$ large enough, and, consequently, it is enough to concentrate on the first term. We estimate

$$
\left|E_{j k} \cap U_{j k}\right| \leq\left|E_{j k} \cap U_{j k} \cap V_{j k}\right|+\left|\complement V_{j k}\right| .
$$


Since the $L^{p}$-norms of the gradients are bounded, there exists $\lambda>0$ such that

$$
\left|\complement V_{j k}\right| \leq \frac{\eta}{3}
$$

and, thus, we can focus attention on the measure of the set $E_{j k} \cap U_{j k} \cap V_{j k}$.

Next, we define

$$
\gamma(x, t)=\inf ((\mathcal{A}(x, t, \xi)-\mathcal{A}(x, t, \zeta)) \cdot(\xi-\zeta)),
$$

where the infimum is taken over the compact set

$$
\left\{(\xi, \zeta) \in \mathbb{R}^{n} \times \mathbb{R}^{n}:|\xi|,|\zeta| \leq \lambda,|\mathcal{A}(x, t, \xi)-\mathcal{A}(x, t, \zeta)| \geq \rho\right\} .
$$

The compactness of the this set was the reason for defining the set $V_{j k}$ above. Due to compactness of the above set, continuity of $\xi \mapsto \mathcal{A}(x, t, \xi)$ and monotonicity of $\mathcal{A}$, it follows that $\gamma(x, t)>0$ for almost every $(x, t) \in Q_{t_{1}, t_{2}}$. By (5.5), we obtain with $\theta=1$ in $Q_{t_{1}, t_{2}}$ that for every $\delta>0$

$$
\begin{aligned}
& \int_{E_{j k} \cap U_{j k} \cap V_{j k}} \gamma(x, t) d x d t \\
& \quad \leq \int_{U_{j k}}\left(\mathcal{A}\left(x, t, \nabla u_{j}\right)-\mathcal{A}\left(x, t, \nabla u_{k}\right)\right) \cdot \nabla\left(u_{j}-u_{k}\right) d x d t \leq C \delta .
\end{aligned}
$$

for $j$ and $k$ large enough. Since $\gamma(x, t)>0$ for almost every $(x, t) \in Q_{t_{1}, t_{2}}$, it follows that

$$
\left|E_{j k} \cap U_{j k} \cap V_{j k}\right| \leq \frac{\eta}{3}
$$

for $\delta$ small enough and $j, k$ large enough. We deduce

$$
\left|\left\{(x, t) \in Q_{t_{1}, t_{2}}:\left|\nabla u_{j}(x, t)-\nabla u_{k}(x, t)\right| \geq \rho\right\}\right| \leq \eta
$$

for large enough $j$ and $k$. Hence $\nabla u_{j}, j=1,2, \ldots$, is a Cauchy sequence with respect to the convergence in measure.

Since the sequence $u_{i}, i=1,2, \ldots$, converges almost everywhere to $u$, it is bounded, and $\nabla u_{i}, i=1,2, \ldots$, is a Cauchy sequence in convergence in measure, it follows that $\nabla u_{i}$ converges to $\nabla u$ in measure. Consequently, there exists a subsequence that converges almost everywhere to $\nabla u$. Observe that the above reasoning actually holds for any subsequence, and, thus, the whole sequence converges, i.e. $\nabla u_{i} \rightarrow \nabla u$ almost everywhere as $i \rightarrow \infty$.

Next, by the continuity of $\xi \mapsto \mathcal{A}(x, t, \xi)$, we obtain that $\mathcal{A}\left(x, t, \nabla u_{i}\right)$, $i=1,2, \ldots$, converges to $\mathcal{A}(x, t, \nabla u)$ almost everywhere. Since $\mathcal{A}\left(x, t, \nabla u_{i}\right)$ is uniformly bounded in $L^{p /(p-1)}$, it follows by Hölder's inequality (cf. Corollary 5.7) that $\mathcal{A}\left(x, t, \nabla u_{i}\right)$ converges to $\mathcal{A}(x, t, \nabla u)$ in $L^{q}$ for all $1 \leq q<$ $p /(p-1)$.

Collecting the facts, both terms in the definition of a weak supersolution converge to right limits, proving the assertion.

Remark 5.6. The assumption $-M \leq u_{i} \leq M$ in the previous theorem can be replaced with the assumption that the norms of the gradients of $u_{i}$ are uniformly bounded. This implies that, if $u \in L^{p}\left(t_{1}, t_{2}, W^{1, p}(Q)\right)$, whenever $Q_{t_{1}, t_{2}}=Q \times\left(t_{1}, t_{2}\right) \Subset \Xi$, and $\min (u, k)$ is a weak supersolution for every $k \in \mathbb{R}$, then also $u$ is a weak supersolution.

Note that the proof implies the strong convergence for the sequence of gradients in $L^{q}$, for all $q<p$. 
Corollary 5.7. Let $\Xi$ be an open set in $\mathbb{R}^{n} \times \mathbb{R}$. Suppose that $u_{i}$, $i=$ $1,2, \ldots$ is a sequence of supersolutions in $\Xi$ such that $-M \leq u_{i} \leq M$ and $u_{i}$ converges to $u$ almost everywhere in $\Xi$. Then

$$
\nabla u_{i} \rightarrow \nabla u \quad \text { in } \quad L^{q}\left(Q_{t_{1}, t_{2}}\right) \quad \text { for every } \quad Q_{t_{1}, t_{2}} \Subset \Xi \text { and } 1 \leq q<p .
$$

Proof. First, write

$$
\begin{aligned}
\int_{Q_{t_{1}, t_{2}}}\left|\nabla u_{j}-\nabla u_{k}\right|^{q} d x d t & =\int_{\left\{\left|\nabla u_{j}-\nabla u_{k}\right|<\delta\right\} \cap Q_{t_{1}, t_{2}}}\left|\nabla u_{j}-\nabla u_{k}\right|^{q} d x d t \\
& +\int_{\left\{\left|\nabla u_{j}-\nabla u_{k}\right| \geq \delta\right\} \cap Q_{t_{1}, t_{2}}}\left|\nabla u_{j}-\nabla u_{k}\right|^{q} d x d t .
\end{aligned}
$$

We immediately observe that the first term on the right hand side can be made small by choosing $\delta$ small enough. The second term on the right hand side can be made small since $\nabla u_{i}$ converges in measure and the norms of the gradients are uniformly bounded. Indeed, we employ Hölder's inequality and estimate

$$
\begin{aligned}
& \int_{\left\{\left|\nabla u_{j}-\nabla u_{k}\right| \geq \delta\right\} \cap Q_{t_{1}, t_{2}}}\left|\nabla u_{j}-\nabla u_{k}\right|^{q} d x d t \\
& \leq\left|\left\{\left|\nabla u_{j}-\nabla u_{k}\right| \geq \delta\right\} \cap Q_{t_{1}, t_{2}}\right|^{(p-q) / p}\left(\int_{Q_{t_{1}, t_{2}}}\left|\nabla u_{j}-\nabla u_{k}\right|^{p} d x d t\right)^{q / p} .
\end{aligned}
$$

Furthermore, the definition of the weak gradient and weak convergence of $u_{i}, i=1,2, \ldots$ show that the limit is $\nabla u$. This proves the assertion.

Next we show that a bounded $\mathcal{A}$-superparabolic function is a supersolution.

Theorem 5.8. Let $\Xi$ be an open set in $\mathbb{R}^{n} \times \mathbb{R}$. Suppose that $u$ is $\mathcal{A}$ superparabolic and $u$ is locally bounded or $u \in L^{p}\left(t_{1}, t_{2}, W^{1, p}(Q)\right)$ whenever $Q_{t_{1}, t_{2}} \Subset \Xi$. Then $u$ is a weak supersolution.

Proof. Since $u$ is $\mathcal{A}$-superparabolic, also $u_{k}=\min (u, k), k=1,2, \ldots$, are $\mathcal{A}$-superparabolic. By the lower semicontinuity of $u_{k}$, there exists, for every $k$, a sequence of continuous functions $\psi_{j}, j=1,2, \ldots$ such that

$$
\psi_{1} \leq \psi_{2} \leq \cdots \leq u_{k}
$$

and $\psi_{j} \rightarrow u_{k}$ pointwise as $j \rightarrow \infty$.

In order to show that $u_{k}$ is a weak supersolution in $\Xi$, it suffices to show that $u_{k}$ is a weak supersolution in all the space-time boxes $Q_{t_{1}, t_{2}} \Subset \Xi$. To this end, choose $Q_{t_{1}, t_{2}} \Subset \Xi$ and let $v_{j}$ solve the obstacle problem in $Q_{t_{1}, t_{2}}$ with the obstacle $\psi_{j}$, see Theorem 2.8. The function $v_{j}$ is continuous and bounded in $\bar{Q}_{t_{1}, t_{2}}$ and, in the open set

$$
U=\left\{(x, t) \in Q_{t_{1}, t_{2}}: v_{j}>\psi_{j}\right\}
$$

$v_{j}$ is an $\mathcal{A}$-parabolic function. Because $v_{j}=\psi_{j}$ on $\partial_{p} Q_{t_{1}, t_{2}}$ and $v_{j}$ as well as $\psi_{j}$ are continuous, it follows that $v_{j}=\psi_{j}$ on $\partial U$ except possibly when $t=t_{2}$. Thus, by Corollary 4.6, we conclude that $u_{k} \geq v_{j}$. Consequently, $\psi_{j} \leq v_{j} \leq$ $u_{k}$ and $v_{j}$ converges pointwise to $u_{k}$ in all space-time boxes $Q_{t_{1}, t_{2}} \Subset \Xi$. According to Theorem 5.3, the function $u_{k}$ is a weak supersolution in $\Xi$. 
Furthermore, Theorem 5.3 and Remark 5.6 also imply that $u=\lim _{k \rightarrow \infty} u_{k}$ is a weak supersolution. This finishes the proof.

\title{
REFERENCES
}

[1] H. W. Alt And S. Luckhaus, Quasilinear elliptic-parabolic differential equations, Math. Z., 183 (1983), pp. 311-341.

[2] G. I. BAREnBlatt, On self-similar motions of a compressible fluid in a porous medium, Akad. Nauk SSSR. Prikl. Mat. Meh., 16 (1952), pp. 679-698.

[3] L. Boccardo, A. Dall'Aglio, T. Gallouët, and L. Orsina, Nonlinear parabolic equations with measure data, J. Funct. Anal., 147 (1997), pp. 237-258.

[4] E. DiBenedetto, Degenerate parabolic equations, Universitext, Springer-Verlag, New York, 1993.

[5] E. DiBenedetto and M. A. Herrero, On the Cauchy problem and initial traces for a degenerate parabolic equation, Trans. Amer. Math. Soc., 314 (1989), pp. 187-224.

[6] E. DiBenedetto, J. M. Urbano, And V. Vespri, Current issues on singular and degenerate evolution equations, in Evolutionary equations. Vol. I, Handb. Differ. Equ., North-Holland, Amsterdam, 2004, pp. 169-286.

[7] S. Granlund, P. Lindqvist, And O. Martio, Conformally invariant variational integrals, Trans. Amer. Math. Soc., 277 (1983), pp. 43-73.

[8] P. Juntinen, P. Lindqvist, and J. J. Manfredi, On the equivalence of viscosity solutions and weak solutions for a quasi-linear equation, SIAM J. Math. Anal., 33 (2001), pp. 699-717 (electronic).

[9] T. Kilpeläinen And P. LindQvist, On the Dirichlet boundary value problem for a degenerate parabolic equation, SIAM J. Math. Anal., 27 (1996), pp. 661-683.

[10] J. Kinnunen AND P. LindQVist, Summability of semicontinuous supersolutions to a quasilinear parabolic equation, Ann. Sc. Norm. Super. Pisa Cl. Sci. (5), 4 (2005), pp. 59-78.

[11] _ Pointwise behaviour of semicontinuous supersolutions to a quasilinear parabolic equation, Ann. Mat. Pura Appl. (4), 185 (2006), pp. 411-435.

[12] R. Korte And T. KuUsi And J. SilJAnder, Obstacle problem for nonlinear parabolic equations, J. Differential Equations, 246 (2009), no. 9, pp. 3668-3680.

[13] P. Lindqvist and J. J. MAnfredi, Viscosity supersolutions of the evolutionary pLaplace equation, Differential Integral Equations, 20 (2007), no. 11, pp. 1303-1319.

[14] J.-L. Lions, Quelques méthodes de résolution des problèmes aux limites non linéaires, Dunod, 1969.

[15] R. E. ShowALter, Monotone operators in Banach space and nonlinear partial differential equations, Volume 49 of Mathematical Surveys and Monographs, American Mathematical Society, Providence, 1997.

\author{
RIIKKA KORTE \\ Department of Mathematics and Statistics \\ UNIVERSITY OF HELSINKI \\ P.O. Box 68, 00014 University of Helsinki, \\ HELSinki, Finland \\ riikka.korte@helsinki.fi
}

Tuomo KuUsi

Institute OF MAThematics

Helsinki University of TeChNOLOGY

P.O. BoX 1100, 02015 TKK,

EsPoo, Finland

tuomo.kuusi@tkk.fi 
Mikko Parviainen

Institute of Mathematics

HELSINKI UNIVERSITY OF TECHNOLOGY

P.O. Box 1100, 02015 TKK,

EsPoo, Finland

mikko.parviainen@tkk.fi 